\title{
Acute transaminitis as an atypical presentation of Graft-versus-Host Disease following Hematopoietic Stem Cell Transplantation
}

\author{
Resham Ramkissoon*1, Akshay Shetty ${ }^{2}$, Adam Doyle ${ }^{2}$, Oyedele A. Adeyi ${ }^{3}$, Keyur Patel ${ }^{2}$ \\ ${ }^{1}$ Dartmouth-Hitchcock Medical Center, Department of Internal Medicine, USA \\ ${ }^{2}$ University of Toronto/University Health Network, Division of Gastroenterology, Canada \\ ${ }^{3}$ University of Toronto/University Health Network, Laboratory Medicine Program, Canada
}

Received: February 28, 2019

Accepted: March 18, 2019

Online Published: March 25, 2019

DOI: $10.5430 /$ crcp.v6n1p1

URL: https://doi.org/10.5430/crcp.v6n1p1

\begin{abstract}
Graft versus Host Disease (GVHD) can present with mucocutaneous, gastrointestinal and hepatic manifestations, specifically a cholestatic transaminitis. Rarely, some cases can present with only a hepatocellular transaminitis. Our patient presented with an acute hepatitis on day +90 post-hematopoietic stem cell transplant, without other overt manifestations of GVHD. The initial work up was negative for a viral etiology or causative drug, and the patient's transaminases continued to rise. On day +96, an erythematous rash appeared with biopsy indicating lymphocytic and eosinophilic infiltrates concerning for cutaneous GVHD. Subsequently, a liver biopsy was obtained, and showed marked ductopenia with cholestasis, consistent with hepatic GVHD.
\end{abstract}

Key Words: Transaminitis, Hepatitis, Graft-versus-Host Disease, Hematopoietic Stem Cell Transplant

\section{INTRODUCTION}

Graft-versus-Host Disease (GVHD) is a multi-system disorder seen in $\geq 50 \%$ of long-term survivors following an allogeneic Hematopoietic Stem Cell Transplant (HSCT). ${ }^{[1,2]}$ Acute GVHD often presents within 100 days following a HSCT, whereas chronic GVHD presents after 100 days. Chronic GVHD can be unpredictable as it can present de novo, after the resolution, or as an extension of acute GVHD. $^{[3]}$

The pathophysiology of GVHD starts with the leukocytes, transplanted from an unmatched or non-identical donor, recognizing the transplant recipient as a foreign entity, which triggers an inflammatory reaction. ${ }^{[4]}$ Affected organs in- clude the skin, eyes, salivary glands, gastrointestinal lumen, and liver. Hepatic GVHD typically presents as a cholestatic transaminitis secondary to hepatobiliary injury; with elevated Alkaline Phosphatase (ALP), hyperbilirubinemia, and hepatomegaly. Elevations in Alanine Aminotransferase (ALT) and Aspartate Aminotransferase (AST) are usually mild and delayed in the clinical timeline. Additionally, bilirubin is used to assess the severity and monitor progression of GVHD. ${ }^{[5,6]}$ The mechanism by which GVHD presents with a cholestatic, rather than hepatocellular, pattern is not fully understood but studies contend the bile duct is the primary target of disease. Studies suggest that overexpression of major histocompatibility complex-I antigens in hepatocytes, cytokine/T-cell interactions, and apoptosis induced by

\footnotetext{
*Correspondence: Resham Ramkissoon; Email: resham.a.ramkissoon@ hitchcock.org; Address: Dartmouth Hitchcock Medical Center, Department of Internal Medicine, One Medical Center Drive, Lebanon, New Hampshire, 03756-0000, USA.
} 
Fas-Fas ligands leads to this distinction. ${ }^{[7-9]}$

Histopathology is essential for establishing a diagnosis of GVHD. This is especially important if there are any atypical features, confounding infections, or potential drug toxicities. Without histology to confirm the diagnosis, patients may erroneously receive treatment. A survey of referrals to John Hopkins for consultation regarding chronic GVHD noted that $7 \%$ of patients did not have diagnostic biopsies before starting treatment, and active chronic GVHD was incorrectly diagnosed and treated before the referral was placed. ${ }^{[10]}$ Biopsies have an enormous value in establishing the diagnosis of GVHD however the utility of repeat biopsies to assess treatment response has yet to be determined. Furthermore, screening biopsies in asymptomatic patients, who are still receiving immunosuppressive therapy, is controversial. Asymptomatic patients with a positive biopsy are not considered to have GVHD. ${ }^{[2]}$

Rare variants of hepatic GVHD have been reported following myeloablative HCSTs in a series of three case reports. ${ }^{[11-13]}$ We herein describe a case of acute transaminitis on day +90 following a second allogeneic HSCT for secondary graft failure in a 20-year-old man with acute lymphoblastic leukemia (ALL). This was initially thought to be an acute viral hepatitis given the presenting clinical picture. However, a subsequent liver biopsy, combined with a skin biopsy of a new rash, revealed findings consistent with chronic GVHD.

\section{CASE PResentation}

A 20-year-old man with T-cell precursor ALL (BCR-ABL+) presented with an acute hepatitis on day +90 after a second A-antigen mismatched, 9/10 unrelated donor allogeneic HSCT for secondary failure of the first graft. Prior to transplant, he received the Dana-Farber Cancer Institute ALL Consortium protocol for chemotherapy. ${ }^{[14]}$ He tolerated this therapy without complication apart from HSV-positive mucositis for which he was successfully treated with acyclovir $400 \mathrm{mg}$ twice a day for 7 days. The patient was conditioned with Ffe, busulphan, and total body irradiation as per the UFBT (200-ATG-PTCY-CSA) protocol. Granulocyte colonystimulating factor (G-CSF) was administered at $10 \mathrm{~g} / \mathrm{kg}$ for 5 days. For GVHD prophylaxis, cyclosporine A ( $3 \mathrm{mg} / \mathrm{kg} /$ day) was started on day -1 . On day +16 , he developed hemorrhagic cystitis and cyclophosphamide was discontinued. On day +35 , chimerism studies showed $98 \%$ engraftment despite ongoing, transfusion-dependent pancytopenia. On day +38 , he developed an erythematous, macular rash over his lower torso. Viral etiologies were excluded, and the rash resolved spontaneously.

On day +90 , he presented with right upper quadrant pain and was found to have an acute transaminitis; serum AST 700U/L, ALT 1,282 U/L, ALP $576 \mathrm{U} / \mathrm{L}$, and total bilirubin $3.5 \mathrm{mg} / \mathrm{dl}$. Abdominal ultrasound showed hepatosplenomegaly without portal or hepatic vein thrombosis. Viral serologies were negative for Hepatitis A, C, E, Varicella Zoster virus (VZV), Cytomegalovirus (CMV), and Herpes Simplex virus (HSV). Hepatitis B virus (HBV) serologies revealed a weakly positive HBV surface antigen, positive HBV surface antibody, and positive total HBV core antibody. The patient received the $\mathrm{HBV}$ vaccination as a one-year-old. Prior $\mathrm{HBV}$ and Hepatitis $\mathrm{C}$ virus (HCV) serologies had been negative in the setting of persistent hypogammaglobulinemia from an immunoglobulin deficiency. Lamivudine $100 \mathrm{mg}$ daily was commenced. Subsequently, HBV DNA was reported as being undetectable and Lamivudine was discontinued.

On day +96 , he developed a pruritic, maculopapular rash on his anterior left thigh. A skin biopsy revealed an interface dermatitis with dyskeratotic cells, epidermal-dermal separation, as well as lymphocytic and eosinophilic infiltrates. A subsequent liver biopsy showed marked ductopenia ( $>80 \%)$, severe hepato-canalicular cholestasis with scarce inflammation or ductular reaction. The cholestasis was associated with prominent hepatocellular injury manifesting as clusters of feathery, degenerated hepatocytes (see Figure 1). The patient was started on methylprednisone $2 \mathrm{mg} / \mathrm{kg} / \mathrm{day} \mathrm{IV}$ and cyclosporine $125 \mathrm{mg}$ IV twice a day. Despite treatment, the patient's condition worsened, and he passed away from a systemic bacterial infection.

\section{Discussion}

We described a young man with T-cell precursor ALL who developed hepatic GVHD presenting as a mixed transaminitis (given the high ALT and AST, as well as ALK and bilirubin). Drug-induced liver injury was excluded after his medications were reviewed and on subsequent liver biopsy. The patient did not receive chemotherapy during this hospitalization. Abdominal ultrasound revealed hepatosplenomegaly without evidence of thrombosis. Viral hepatitis was excluded; VZV, CMV and HSV PCRs returned negative. HAV and HCV serologies returned negative. HBV serologies returned positive for $\mathrm{HBsAg}$ having previously been negative before his transplants. The patient had a history of hypogammaglobulinemia, reducing the sensitivity of these tests. He was treated empirically for a possible reactivation versus acute infection in the setting of multiple transfusions. HBV DNA was undetectable excluding possible occult infection, and repeat $\mathrm{HBV}$ serologies were negative. The skin biopsy of a new rash revealed lympho-eosinophilic infiltration, increasing our pre-test probability for GVHD. This prompted a liver biopsy that proved GVHD. This concern for a confound- 
ing HBV infection highlights the importance of biopsy with histopathology in the diagnosis of GVHD.

In acute GVHD, cutaneous involvement is the most common presenting sign, followed by intestinal and hepatic involvement. Rarely, hepatic manifestations of GVHD can present prior to cutaneous manifestations, which has only been documented in case reports. The characteristic features of acute GVHD are bile duct damage and cholestasis. A case series of acute GVHD describes patients presenting with high transaminases and, following liver biopsies, were found to have bile duct changes without ductopenia and lobar hepatitis with portal inflammation. These features in the case series were markedly more prominent than features commonly seen in acute GVHD. ${ }^{[1-13,15]}$ Chronic GVHD is characterized by bile-duct dystrophy and senescence, ductopenia and portal fibrosis, which is often seen on trichrome stains. ${ }^{[13]}$ We initially suspected this patient had acute GVHD because he presented within 100 days post-transplant; the rash present on the patient's torso may have been the first and earliest manifestation of acute GVHD. However, chronic GVHD that began following the first HSCT cannot be excluded. There is a consensus for the Histopathologic Diagnosis of Chronic
Graft-versus-Host Disease and, based on histological and clinical findings, this patient would fit into the category of chronic GVHD.

This presentation was quite unusual for GVHD, but we postulate the degree of intrahepatic bilirubinostasis accounted for a large part of the hepatocellular injury (see Figure 1). This finding, along with the marked ductopenia, has not been seen in prior case reports. It is possible that another unknown factor contributes to high transaminases in GVHD patients. Our patient was myeloablated prior to the first HSCT, but not the second. Non-myeloablative therapy is reported to be non-inferior to myeloablative therapies in the development of GVHD. Other studies purport a greater risk of chronic GVHD associated with non-ablative therapy. ${ }^{[16,17]}$ Recent discontinuation of immunosuppressive agents has also been implicated in contributing to the onset of GVHD; ${ }^{[13]}$ cyclophosphamide was discontinued after the patient developed hemorrhagic cystitis. However, it is unclear if non-myeloablative therapies and discontinuation of immunosuppressive agents contribute to atypical presentations of GVHD.
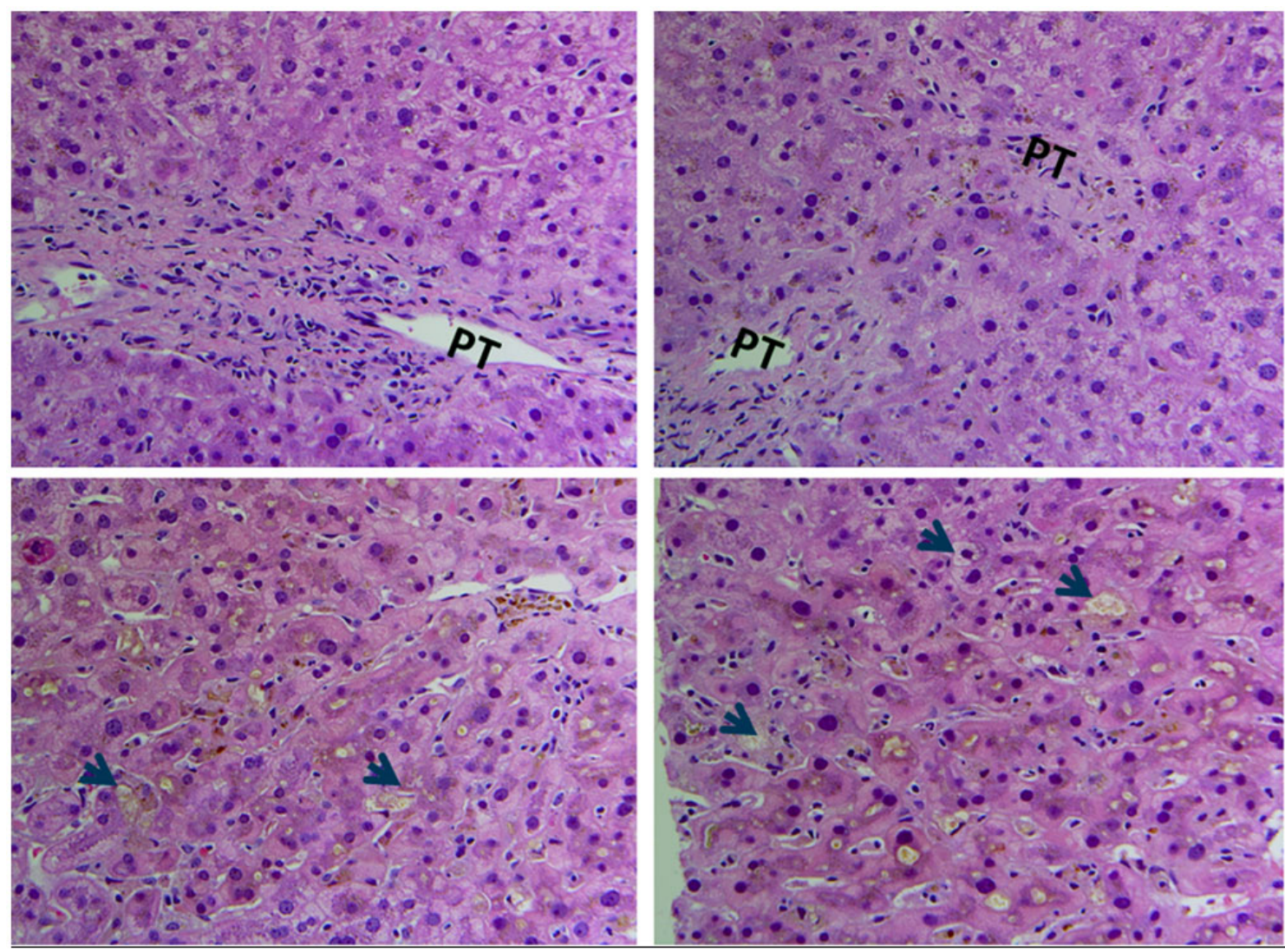

Figure 1. Liver with absent bile ducts in the portal tracts (PT) associated with little to no inflammation and absence of ductular reaction. There is extensive hepatocanalicular cholestasis with secondary damage to hepatocytes (arrows) (Hematoxylin \& eosin stain; original magnification $200 \times$ ). 
To conclude, we report our experience with a case of chronic GVHD following a second allogeneic HSCT, presenting as an acute transaminitis rather than the predominantly cholestatic picture. We postulate this was a consequence of hepatocellular injury from intra-hepatic retention of bile secondary to GVHD-damaged bile ducts. This observation, in combi- nation with prior literature, suggests that GVHD must be included in the differential diagnosis of an acute transaminitis, either cholestatic or hepatocellular, following HSCT.

\section{CONFlicts OF InTEREST Disclosure}

The authors declare that they have no competing interest.

\section{REFERENCES}

[1] McDonald GB, Shulman HM, Wolford JL, et al. Liver disease after human marrow transplantation. Seminars in Liver Disease. 1987; 7 : 210-29. PMid:3317859. https ://doi.org/10.1055/s-2008-1 040578

[2] Shulman HM, Kleiner D, Lee SJ, et al. Histopathologic Diagnosis of Chronic Graft-versus-Host Disease: National Institutes of Health Consensus Development Project on Criteria for Clinical Trials in Chronic Graft-versus-Host Disease: II. Pathology Working Group Report. Biology of Blood and Marrow Transplantation. 2006; 12: 31-47. https://doi.org/10.1016/j.bbmt.2005.10.023

[3] Toubai T, Sun Y, Reddy P. GVHD pathophysiology: is acute different from chronic? Best Practice \& Research: Clinical Hematology. 2008; 21(2): 101-17. https://doi.org/10.1016/j.beha. 200 8.02 .005

[4] Ferrara JL, Yanik G. Acute graft versus host disease: pathophysiology, risk factors, and prevention strategies. Clinical Advances in Hematology \& Oncology. 2005; 3(5): 415-9. Available from: https://www.ncbi.nlm.nih.gov/pubmed/16167015

[5] Atkinson K. Chronic graft-versus-host disease. Bone Marrow Transplant. 1990; 5(2): 69-82. Available from: https://www.ncbi.nlm .nih.gov/pubmed/2178709

[6] Crawford JM. Graft-versus-Host Disease (2nd ed.). New York, NY: Marcel Dekker; 1997.

[7] Ferrara JL, Deeg HJ. Graft-versus-host disease. New England Journal of Medicine. 1991; 324(10): 667-74. PMid:1994250. https: //doi.org/10.1056/NEJM199103073241005

[8] Yasmineh WG, Filipovich AH, Killeen AA. Serum 5'nucleotidase and alkaline phosphatase - highly predictive liver function tests for the diagnosis of graft-versus-host disease in bone marrow transplantation recipients. Transplantation. 1989; 48: 809-14. PMid:2554545 https://doi.org/10.1097/00007890-198911000-00017

[9] Lazaridis KN, Gores GJ, Lindor KD. Ursodeoxycholic acid mechanisms of action and clinical use in hepatobiliary disorders. Journal of Hepatology. 2001; 35: 134-46. https ://doi .org/10.1016/s0 $168-8278(01) 00092-7$
[10] Jacobsohn DA, Montross S, Anders V, et al. Clinical importance of confirming or excluding the diagnosis of chronic graft-versus-host disease. Bone Marrow Transplant. 2001; 28: 1047-51. PMid:11781615. https ://doi.org/10.1038/sj.bmt.1703278

[11] Fujii N, Takenaka K, Shinagawa K, et al. Hepatic graft-versus-host disease presenting as an acute hepatitis after allogeneic peripheral blood stem cell transplantation. Bone Marrow Transplant. 2001; 27: 1007-10. PMid:11436113. https ://doi.org/10.1038/sj.bmt .1702997

[12] Malik AH, Collins RH, Saboorian MH, et al. Chronic graft versushost disease after hematopoietic cell transplantation presenting as an acute hepatitis. American Journal of Gastroenterology. 2001; 96: 58890. PMid:11232714. https://doi .org/10.1111/j.1572-0241. 2001.03563. $\mathrm{x}$

[13] Strasser SI, Shulman HM, Flowers ME, et al. Chronic graft-versus host disease of the liver: presentation as an acute hepatitis. Hepatology. 2000; 32: 1265-71. PMid:11093733. https://doi.org/10 .1053/jhep. 2000.20067

[14] Silverman LB, Stevenson KE, O'Brien JE, et al. Long-term results of Dana-Farber Cancer Institute ALL Consortium protocols for children with newly diagnosed acute lymphoblastic leukemia 19852000. Leukemia. 2010; 24(2): 320-334. PMid:20016537. https : //doi.org/10.1038/leu.2009.253

[15] Maeng H, Lee JH, Cheong JW, et al. Chronic Graft-versus-Host Disease of the Liver Presenting as an Acute Hepatitis following Non-myeloablative Hematopoietic Stem Cell Transplantation. International Journal of Hematology. 2004; 79: 501-4. Available from: https://www.ncbi.nlm.nih.gov/pubmed/15239404

[16] Mielcarek M, Martin PJ, Leisenring W, et al. Graft-versus-host disease after nonmyeloablative versus conventional hematopoietic stem cell transplantation. Blood. 2003; 102(2): 756-62. PMid:12663454. https://doi .org/10.1182/blood-2002-08-2628

[17] Chen MH, Chiou TJ, Lin PC, et al. Comparison of myeloablative and nonmyeloablative hematopoietic stem cell transplantation for treatment of chronic myeloid leukemia. International Journal of Hematology. 2007; 86(3): 275-81. https://doi.org/10.1007/BF03 006933 